\title{
Ty1 reverse transcriptase does not read through the proposed 2',5'-branched retrotransposition intermediate in vitro
}

\author{
ELIZABETH D. PRATICO and SCOTT K. SILVERMAN \\ Department of Chemistry, University of Illinois at Urbana-Champaign, Urbana, Illinois 61801, USA
}

\begin{abstract}
2',5'-Branched RNA was recently proposed as a key Ty1 retrotransposition intermediate, for which cleavage by lariat debranching enzyme (Dbr1p) enables reverse transcription to continue synthesizing the complete Ty1 cDNA. Because dbr1 cells can produce substantial Ty1 cDNA despite lacking Dbr1p, the obligatory intermediacy of branched RNA would require that Ty1 reverse transcriptase (RT) can read through the proposed branch site with considerable efficiency. Here we have used deoxyribozyme-synthesized 2',5'-branched RNA corresponding exactly to the proposed Ty1 branch site for a direct test of this read-through ability. Using an in vitro assay that incorporates all components known to be required for Ty1 cDNA synthesis (including the TyA chaperone protein), Ty1 RT can elongate up to the branch site. Strand transfer from the $2^{\prime}$-arm to the $3{ }^{\prime}$-arm of the branch is observed when the Ty1 RT is RNase $\mathrm{H}^{+}$(i.e., wild-type) but not when the Ty1 RT is RNase $\mathrm{H}^{-}$. When elongating from either the $2^{\prime}$-arm or the $3^{\prime}$-arm, Ty1 RT reads through the branch site with $\leq 0.3 \%$ efficiency. This is at least 60 -fold lower than would be necessary to explain in vivo Ty1 cDNA synthesis in dbr1 cells, because others have reported $18 \%$ cDNA synthesis relative to wild-type cells. Our finding that Ty1 RT cannot efficiently read through the proposed Ty 1 branch site is inconsistent with the hypothesis that branched RNA is an obligatory Ty1 retrotransposition intermediate. This suggests that Dbr1p acts as other than a $2^{\prime}, 5^{\prime}$-phosphodiesterase during Ty1 retrotransposition.
\end{abstract}

Keywords: Ty1 retrotransposition; reverse transcriptase; read-through; deoxyribozyme; branched RNA; debranching enzyme

\section{INTRODUCTION}

The lariat debranching enzyme Dbrlp was first identified in 1991 by a genetic screen in Saccharomyces cerevisiae for cellular proteins that participate in retrotransposition of the Ty1 element (Chapman and Boeke 1991). The contribution of Dbrlp to the retrotransposition mechanism has been challenging to elucidate. A recent and controversial explanation (Cheng and Menees 2004) is that Dbrlp is required for debranching of a $2^{\prime}, 5^{\prime}$-branched RNA intermediate, which is the template for minus-strand cDNA synthesis. The evidence for this branched RNA structure was indirect (i.e., the branched RNA was not directly detected) (Perlman and Boeke 2004), and others have subsequently challenged the methodology used to support the intermediacy of branched RNA (Coombes and Boeke 2005).

Reprint requests to: Scott K. Silverman, Department of Chemistry, University of Illinois at Urbana-Champaign, 600 South Mathews Avenue, Urbana, IL 61801, USA; e-mail: scott@scs.uiuc.edu; fax: (217) 244-8024.

Article published online ahead of print. Article and publication date are at http://www.rnajournal.org/cgi/doi/10.1261/rna.629607.
Mutant $d b r 1$ cells lacking Dbr1p nevertheless can produce substantial Ty1 cDNA (Karst et al. 2000; Griffith et al. 2003; Mou et al. 2006) and have a substantial Tyl transposition frequency when compared with wild-type cells (Salem et al. 2003). As noted by Perlman and Boeke (2004) in their commentary on the original paper (Cheng and Menees 2004), if the hypothesis of an obligatory Tyl branched RNA intermediate is correct, then it must be the case that "the Ty1 RT may occasionally yield full-length cDNA by reading through the $2^{\prime}, 5^{\prime}$-branch (perhaps without strand transfer)." This postulated branch read-through ability by Ty1 RT may seem unlikely, given that mapping of $2^{\prime}, 5^{\prime}$-branch sites by RTs — which halt at the branch sites-is well known from early work on RNA splicing (Domdey et al. 1984; Rodriguez et al. 1984; Zeitlin and Efstratiadis 1984). However, both Cheng and Menees (2004) as well as Perlman and Boeke (2004) suggested that read-through of the proposed Tyl branch by Ty1 RT is plausible on the basis of several experiments with unrelated RTs, which showed that in at least some instances RTs can eventually read through either a 2',5'-branch site (Vogel et al. 1997; Tuschl et al. 1998; 
Vogel and Borner 2002; Salem et al. 2003) or a linear 2'-5' linkage (Lorsch et al. 1995). This situation brings into sharp focus a key question: Is Tyl RT actually capable of reading through the proposed Tyl RNA branch site? Because the proposed Tyl branched RNA has never been directly detected nor has it been available via artificial synthesis, answering this key question by a direct assay of Ty1 readthrough ability has until now been impossible.

We have recently reported deoxyribozymes (DNA enzymes) that synthesize 2',5'-branched RNA (Wang and Silverman 2003b, 2005; Coppins and Silverman 2005; Pratico et al. 2005; Zelin et al. 2006). One of these deoxyribozymes, 6CE8, is capable of synthesizing branched RNA that has a branch-site uridine nucleotide (Pratico et al. 2005), such as that found in the proposed Tyl branch. We previously reported a strategy to allow synthesis of branched RNA for which both oligonucleotide strands emerging in the $3^{\prime}$ direction from the branch-site nucleotide have the same sequence (Pratico et al. 2005). This is the situation for the proposed Tyl branched RNA, in which the " $R$ " portion of the long terminal repeat (LTR) that is characteristic of LTR-retrotransposons (Boeke et al. 1985; Havecker et al. 2004) composes both of these strands. In this report, we have used synthetic 2',5'-branched RNA created by the 6CE8 deoxyribozyme and corresponding exactly to the proposed Tyl branch-site RNA sequence for directly testing the ability of Tyl RT to read through the proposed branch in vitro. Our assay system incorporates all components known to be required for Ty1 cDNA synthesis, including the TyA chaperone protein (Cristofari et al. 2002). Whereas elongation up to the branch point and strand transfer from the $2^{\prime}$-arm to the $3^{\prime}$-arm are confirmed with the synthetic branched RNA as template, the Ty1 RT is found to be incapable of efficiently reading through the $2^{\prime}, 5^{\prime}$-branch when elongating from either the $2^{\prime}$-arm or the $3^{\prime}$-arm. This does not support any mechanism for Ty1 cDNA synthesis that proposes branched RNA as an obligatory Tyl retrotransposition intermediate.

\section{RESULTS}

\section{In vitro assay system for Ty1 cDNA synthesis}

An assay for Tyl cDNA synthesis has previously been established in vitro (Fig. 1A; Cristofari et al. 2002). As the template, the 5' Ty1 RNA and 3' Tyl RNA interact solely by noncovalent interactions termed the CYC pairing (Cristofari et al. 2002). The remaining components of this assay system are the Tyl reverse transcriptase (Ty1 RT), the TyA Gag-like chaperone protein, which is included to the extent of one protein molecule for every 2-10 nt of RNA template, and the $\mathrm{tRNA}^{\mathrm{iMet}}$ primer for initiating cDNA synthesis, which binds to a specific region of the $5^{\prime}$ Ty1 RNA (Bolton et al. 2005). For practical reasons we used the full-length TyA protein (476 amino acids) rather than the peptide fragment (103 amino acids) reported by Darlix and coworkers (Cristofari et al. 2002). As a control, experiment A (Fig. 2) demonstrates the synthesis of both strong-stop cDNA (ss-cDNA) and strand-transfer cDNA (st-cDNA) using the two noncovalently associated $5^{\prime}+3^{\prime}$ Tyl RNAs as template. The first product of reverse transcription is ss-cDNA, named as such because elongation stops upon reaching the $5^{\prime}$ terminus of the RNA. The st-cDNA is a secondary product formed by continued elongation after transfer of the growing DNA strand to the second LTR. In experiment $A$, although synthesis of full-length st-cDNA is not completely efficient (i.e., a shorter ss-cDNA is also present), the $\sim 30 \%$ efficiency of st-cDNA synthesis found in our hands is comparable to the $40 \%$ observed by others (Cristofari et al. 2002). The efficiency of st-cDNA synthesis in this control experiment depends strongly on the included amount of TyA chaperone protein, similar to observations by others (Cristofari et al. 2002).

\section{Synthesis of 2',5'-branched RNA comprising the key portions of the proposed Ty1 branched intermediate}

To enable a direct in vitro test of the viability of $2^{\prime}, 5^{\prime}$ branched RNA as the Tyl retrotransposition intermediate, we used the 6CE8 deoxyribozyme to synthesize the necessary $2^{\prime}, 5^{\prime}$-branched RNA. This required two key changes to the RNA ligation procedure as used in our previous report (Pratico et al. 2005). First, the 5' Tyl RNA was $580 \mathrm{nt}$ (Cristofari et al. 2002) rather than 202 nt, because preliminary assays established that the longer version of the 5' Ty1 RNA is required for proper nucleoprotein formation (data not shown). Second, we included a disruptor DNA oligonucleotide during 6CE8 ligation, to sequester secondary structure within the relatively long 5' Ty1 RNA. With these procedural modifications, the branch corresponding exactly to the proposed Tyl branched RNA was prepared as a template for assessing its ability to support ss-cDNA and st-cDNA synthesis by Tyl RT.

\section{Testing the proposed Ty1 branched RNA as a template for synthesis of strand-transfer cDNA}

Using the in vitro assay system described above, the synthetic branched RNA was tested as a Tyl RT template to determine if any st-cDNA was formed, which would indicate that Ty1 RT had read through the branch site. A depiction of the assay is shown in Figure 1B. Alongside the positive control of the noncovalently associated $5^{\prime}+3^{\prime}$ Ty 1 RNAs, with the branched RNA as template no st-cDNA was observed and only ss-cDNA was formed (Fig. 2, experiment B).

This failure to synthesize st-cDNA could be explained in one of two ways. (1) The ss-cDNA is synthesized by elongation along the $2^{\prime}$-arm of the branched RNA until the branch site is reached. At that point, elongation halts 


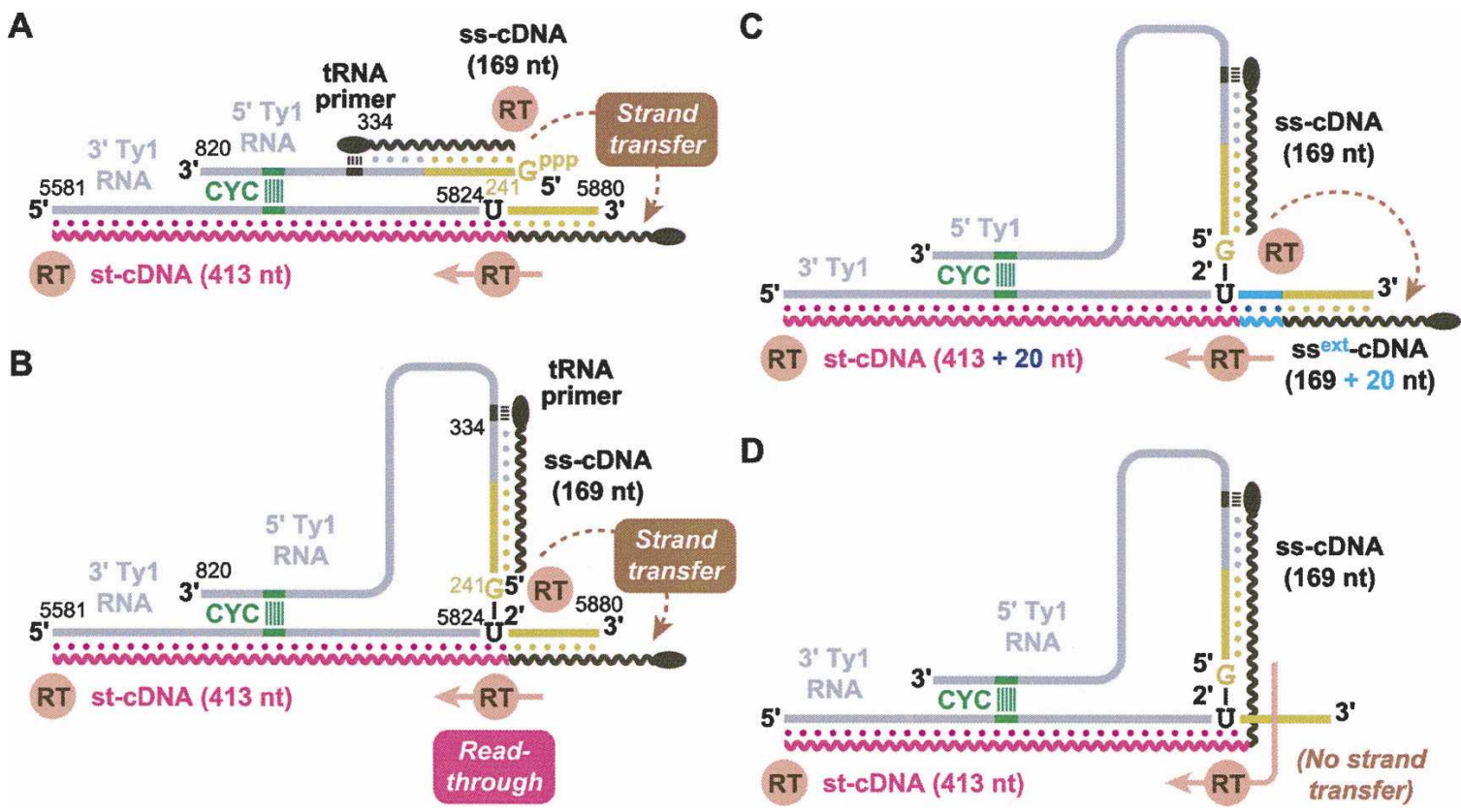

FIGURE 1. Possible structures of the Tyl RNAs during the minus-strand cDNA synthesis step of retrotransposition. $(A)$ The accepted positive control for Tyl retrotransposition, consisting of the $5^{\prime}+3^{\prime}$ Tyl RNAs that interact by noncovalent Watson-Crick base pairing denoted CYC (Cristofari et al. 2002). Both strong-stop cDNA (ss-cDNA) and strand-transfer cDNA (st-cDNA) are depicted as products. The ss-cDNA arises from elongation up to the point where strand transfer occurs; the st-cDNA arises after strand transfer of the ss-cDNA followed by continued elongation. (B) The proposed 2',5'-branched RNA intermediate (Cheng and Menees 2004), which was hypothesized to be the cDNA template in mutant $d b r 1$ cells that lack debranching enzyme (Dbrlp). The ss-cDNA would be synthesized by elongation up to the branch site. Synthesis of stcDNA in $\mathrm{dbrl}$ cells could involve strand transfer from the $2^{\prime}$-arm to the $3^{\prime}$-arm, followed by elongation from the $3^{\prime}$-arm with read-through of the branch site. (C) The proposed 2',5'-branched RNA with an additional, unnatural 20-nt insert (blue) at the branch site. Strand transfer followed by elongation to the branch site (but not read-through to form st-cDNA) would result in an extended ss ${ }^{\text {ext }}$-cDNA that is longer than the ss-cDNA formed in $B$ by the length of the insert. Because strand transfer does not change the length of the cDNA, the insert allows experimental detection of strand transfer if subsequent read-through does not occur. $(D)$ As an alternative to the read-through event depicted in $B$, read-through of the branch site could occur by elongation from the $2^{\prime}$-arm, without prior strand transfer of the cDNA from the $2^{\prime}$-arm to the $3^{\prime}$-arm. Nucleotide numbering in $A$ and $B$ is as used previously (Boeke et al. 1988).

and neither strand transfer nor read-through occurs. (2) The ss-cDNA is synthesized and strand transfer then occurs to the $3^{\prime}$-arm, but subsequent read-through does not take place. These two explanations both invoke a failure of readthrough, in (1) by starting from the $2^{\prime}$-arm of the branch as template and in (2) by starting from the $3^{\prime}$-arm. The two explanations cannot be distinguished solely on the basis of experiment $\mathrm{B}$ because strand transfer does not change the size of the ss-cDNA, and in neither explanation is the ss-cDNA elongated further.

\section{Strand transfer using a branched RNA template containing a small insert near the branch site}

By inserting an additional 20 nucleotide (nt) portion of RNA between the branch site and the 3 '-arm (Fig. 1C), we distinguished the two explanations outlined above for observing only ss-cDNA in experiment B. If strand transfer from the $2^{\prime}$-arm to the $3^{\prime}$-arm does not occur on the insertcontaining branched RNA, then the ss-cDNA product will be the same size as in the absence of the RNA insert. However, if strand transfer occurs, then the cDNA should subsequently be elongated for a short distance using the insert as a template until the branch site is reached, thereby extending the length of the ss-cDNA by $20 \mathrm{nt}$ (Fig. 1, denoted $s s^{\text {ext }}$-cDNA). Strand transfer is fully expected because pauses during reverse transcription are known to induce template switching (Wu et al. 1995; Roda et al. 2002, 2003; Lanciault and Champoux 2006). Indeed, a substantial amount of the $\mathrm{ss}^{\mathrm{ext}}{ }_{-} \mathrm{cDNA}$ product was observed (Fig. 2, experiment C), indicating that strand transfer did occur. The presence of the insert could conceivably enable strand transfer, which in this scenario would not occur at all without the insert. Nevertheless, the simplest explanation of the data is that strand transfer does take place with the proposed branched RNA as template but that readthrough does not subsequently occur from the $3^{\prime}$-arm of the branch.

\section{Using mutant RNase $\mathrm{H}^{-}$Ty1 RT to test for read-through when elongating from the 2 '-arm}

The above data demonstrate that strand transfer of the elongated cDNA occurs from the $2^{\prime}$-arm to the $3^{\prime}$-arm of 


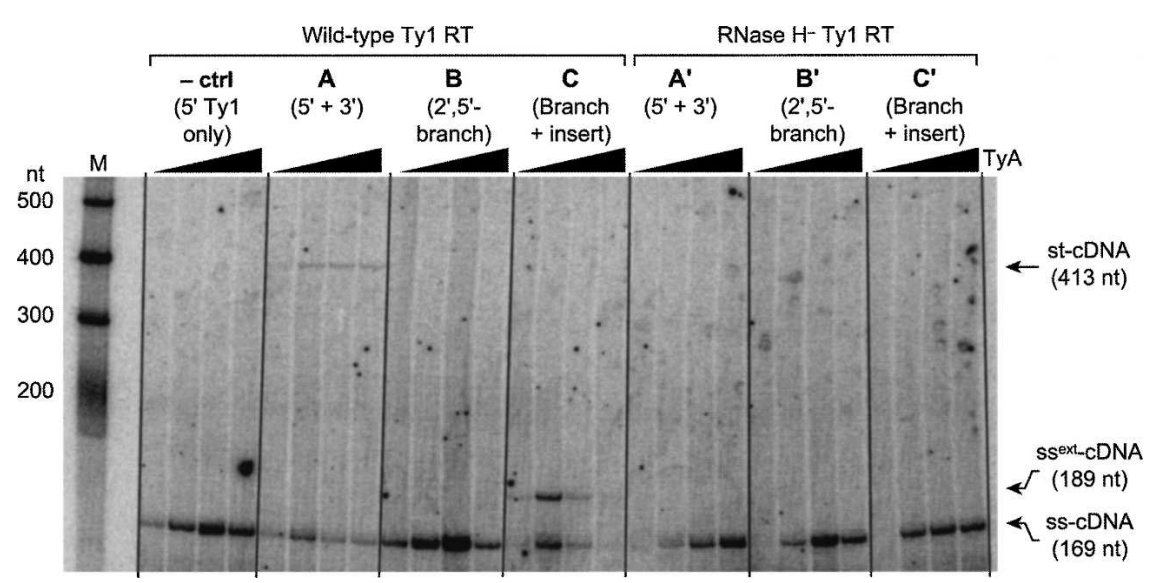

FIGURE 2. Experimental tests of minus-strand cDNA synthesis using possible Tyl RNA structures as templates. The negative control experiment ( $-\mathrm{ctrl})$ used only the $5^{\prime}$ Tyl RNA as the template. Experiments $\mathrm{A}-\mathrm{C}$ and $\mathrm{A}^{\prime}-\mathrm{C}^{\prime}$ use the corresponding RNAs of Figure $1, A-C$, as the templates $(t=60 \mathrm{~min})$. Control experiments $\mathrm{A}$ and $\mathrm{A}^{\prime}$ demonstrate that noncovalently associated $5^{\prime}+3^{\prime}$ Tyl RNAs are sufficient for substantial generation of st-cDNA and that the mutant RNase $\mathrm{H}^{-}$Tyl RT does not permit strand transfer, therefore leading only to ss-cDNA. See text for description of the other experiments. Within each experiment, the four lanes indicate TyA chaperone protein included in the amount of $0,0.1,0.2$, or 0.4 equivalents per Ty1 RNA nucleotide. The sharp dependence of cDNA synthesis on amount of TyA has been observed previously in the control system that uses $5^{\prime}+3^{\prime}$ Tyl RNAs (Cristofari et al. 2002). (M) RNA ladder marker.

the branched RNA, but that Tyl RT cannot subsequently read through the branch site by extending the cDNA starting from the $3^{\prime}$-arm. Because strand transfer is not necessarily $100 \%$ efficient, some read-through could potentially occur by elongation from the 2 '-arm without strand transfer (Fig. 1D). As noted by Perlman and Boeke (2004), full-length st-cDNA synthesis would be enabled by elongation with read-through past the branch point either with or without strand transfer. To test read-through from the $2^{\prime}$-arm without any strand transfer, we assayed the proposed branched RNA in conjunction with a mutant Tyl RT that is known to be defective in the RNase $\mathrm{H}$ endonuclease activity required for degradation of the $5^{\prime}$ Tyl RNA near the branch site ("RNase $\mathrm{H}^{-}$Ty1 RT") (Wilhelm et al. 2001; Cristofari et al. 2002). Degradation of the $5^{\prime}$ Tyl RNA by the RNase $\mathrm{H}$ activity of Tyl RT is a prerequisite for strand transfer of the growing cDNA from the $5^{\prime}$ Tyl RNA to the 3' Ty1 RNA (Cristofari et al. 2002). Therefore, using RNase $\mathrm{H}^{-}$Ty1 RT prevents the elongating cDNA product from undergoing strand transfer from the $2^{\prime}$-arm to the $3^{\prime}$-arm. Consistent with this, only ss-cDNA and no st-cDNA is observed in our hands with the $5^{\prime}+3^{\prime}$ Tyl control substrates and RNase $\mathrm{H}^{-}$Ty1 RT (Fig. 2, experiment $\mathrm{A}^{\prime}$ ), as reported (Cristofari et al. 2002).

Using the proposed Tyl branched RNA as template, the RNase $\mathrm{H}^{-}$Tyl RT led to only ss-cDNA and no st-cDNA (Fig. 2, experiment $B^{\prime}$ ). Therefore, the Ty1 reverse transcriptase was unable to read through the branch site when elongating from the $2^{\prime}$-arm, just as it could not read through from the $3^{\prime}$-arm (Fig. 2, experiment B). For completeness, the failure of RNase $\mathrm{H}^{-}$Tyl $\mathrm{RT}$ to engage in strand transfer was confirmed by using the branched RNA template containing the 20-nt insert (Fig. 2, experiment $\left.C^{\prime}\right)$. As expected, the longer ss ${ }^{\text {ext }}$. cDNA elongation product that had been formed using the wild-type Ty1 RT (by continued elongation along the $3^{\prime}$-arm up to the branch point) was not formed with the mutant Tyl RT. Instead, we observed only the shorter ss-cDNA product from elongation along the $2^{\prime}$ arm without strand transfer.

\section{Quantifying the st-cDNA produced by read-through of the branch}

In summary of the above results, no stcDNA was evident as a product of readthrough from either the $2^{\prime}$-arm or the $3^{\prime}$-arm using the proposed branched RNA as the Tyl RT template (Fig. 2). Considering the relatively low signal-tonoise ratio of our gel images, we sought a more quantitative assessment of the amount of st-cDNA that was produced. For this purpose, we excised the portion of the polyacrylamide gel that would contain any st-cDNA product from the proposed branch as template (using st-cDNA from the $5^{\prime}+3^{\prime}$ Tyl RNAs as a migration marker) and performed PCR amplification using appropriate primers. This allowed us to quantify the st-cDNA product relative to the amount produced from the noncovalently associated $5^{\prime}+3^{\prime}$ Tyl RNAs as a positive control template (i.e., we compared st-cDNA synthesis in experiments B and A in Fig. 2). The PCR data indicate that only $\leq 0.3 \%$ of the amount of st-cDNA is produced relative to the control template when wild-type Ty1 RT is used to produce cDNA from the branch (Fig. 3). For comparison, two groups have independently reported that $d b r 1$ cells produce $\sim 18 \%$ of the Ty1 cDNA that is formed in wildtype cells (Griffith et al. 2003; Mou et al. 2006). Therefore, the amount of st-cDNA that is made from the branch as template is at least $\sim 18 / 0.3=60$-fold short of the amount necessary to explain Tyl cDNA synthesis in dbrl cells. Even less st-cDNA was synthesized using the RNase $\mathrm{H}^{-}$Tyl RT $(<0.05 \%$ relative to control template; Fig. $3 \mathrm{~B}$, “ $X$ ”).

\section{DISCUSSION}

The main goal of our in vitro experiments was to test directly whether or not Tyl RT can read through the proposed Tyl branch site when elongating from either of the two possible oligoribonucleotide strands that are connected to the branch site (i.e., the $3^{\prime}$-arm and $2^{\prime}$-arm; Fig. 1). The hypothesis of an obligatory Ty1 branched RNA 


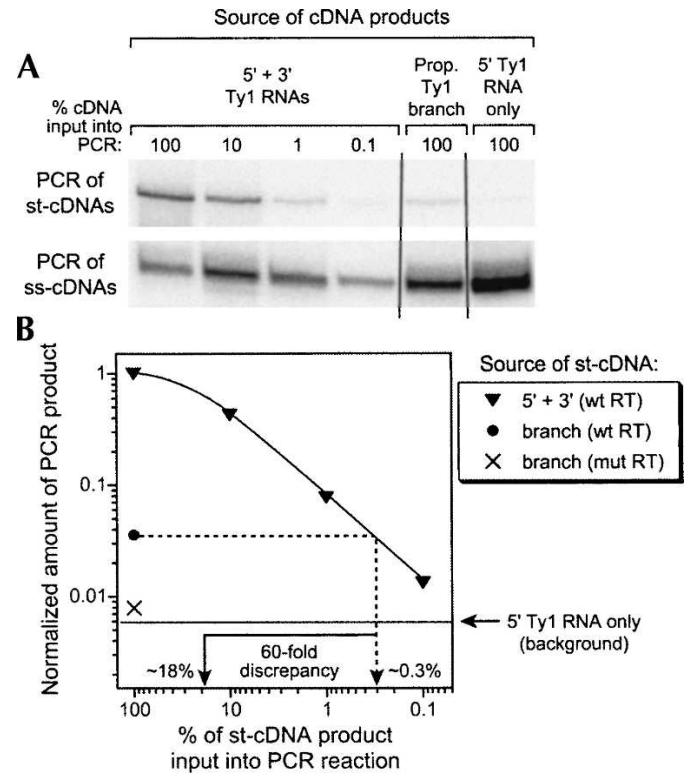

FIGURE 3. PCR to determine the efficiency of st-cDNA synthesis by Tyl RT read-through of the proposed Tyl branch. (A) The st-cDNA and ss-cDNA products from the A, B, and - ctrl experiments of Figure 2 were PCR amplified and analyzed by PAGE. (B) Quantification of the PCR products. The dashed lines show that the amount of st-cDNA produced from the proposed branched RNA is $\leq 0.3 \%$ of the st-cDNA produced from the $5^{\prime}+3^{\prime}$ control Tyl RNAs (using the wildtype Tyl RT in both cases). This contrasts sharply with $\sim 18 \%$ expected (i.e., $\geq 60$-fold discrepancy) if the branched RNA could be used efficiently as a reverse transcription template (Griffith et al. 2003; Mou et al. 2006). The standard deviation was approximately the size of each data point $(n=4$ or 5$)$. The horizontal line denotes the background level of PCR product observed from the $5^{\prime}$ Tyl RNA only, which is a negative control that cannot be a template for st-cDNA synthesis because it lacks the 3' Tyl RNA sequence. The cross marks the amount of PCR product obtained from the st-cDNA synthesized using the branch with the RNase $\mathrm{H}^{-}$mutant Tyl RT, which would perform read-through from the $2^{\prime}$-arm (Fig. 2, experiment $\left.\mathrm{B}^{\prime}\right)$.

intermediate (Cheng and Menees 2004) demands such read-through ability, to explain the production of st-cDNA in dbr1 mutant cells that lack debranching activity by Dbrlp (Perlman and Boeke 2004). However, our results (Fig. 2) show that Tyl RT is incapable of sufficient readthrough to support the branched RNA hypothesis, when tested in vitro with all other components known to be required for st-cDNA synthesis (Ty1 RT, TyA chaperone protein, and $\mathrm{RRNA}^{\mathrm{iMet}}$ primer). The quantitative estimate of an upper limit to st-cDNA synthesis (Fig. 3) indicates that read-through in vitro is about 60 -fold less efficient than required to explain the observed st-cDNA synthesis in dbrl cells. This insufficient level of read-through when tested directly with the proposed Tyl branched RNA in vitro is direct empirical evidence that is inconsistent with the hypothesis that branched RNA is an obligatory Tyl retrotransposition intermediate. All previous evidence against a branched RNA intermediate was indirect, because the proposed branched RNA was unavailable for appropriate positive controls (Coombes and Boeke 2005). Here we provide direct evidence that the proposed branched RNA is not a likely Tyl retrotransposition intermediate because the Ty1 RT cannot perform read-through using the branch as a template.

The data reveal that strand-transfer from the $2^{\prime}$-arm to the $3^{\prime}$-arm can occur on the proposed Tyl branched RNA. Although such strand transfer might generically be expected due to pause-induced template switching (Wu et al. 1995; Roda et al. 2002, 2003; Lanciault and Champoux 2006), explicitly demonstrating strand transfer is important in our system to show that potential readthrough of the branch site is being assayed from both of the possible directions (i.e., from the $2^{\prime}$-arm and $3^{\prime}$-arm). One of the attractive features of the proposed branch was that its covalent connectivity inherently holds together the two oligonucleotide strands that are relevant for strand transfer of the elongating minus-strand cDNA (Cheng and Menees 2004). Alternatively, the CYC pairing in the noncovalent $5^{\prime}+3^{\prime}$ Tyl RNAs (Cristofari et al. 2002) could reasonably enable strand transfer by holding together the necessary strands without requiring a covalent branch. Cheng and Menees (2004) additionally suggested the possibility of base-pairing interactions much closer to the putative branch site; such interactions could hold together the $5^{\prime}$ and $3^{\prime}$ Tyl RNAs (via nucleotides 241245 and $5820-5824)$ as efficiently as a covalent $2^{\prime}, 5^{\prime}$ branch. However, Boeke and coworkers (Bolton et al. 2005) provided evidence that nucleotides 241-247 (which are part of the proposed interaction) are engaged in a different interaction with other nucleotides (504-510) of the 5' Tyl RNA.

In contrast to strand transfer, the putative ability of Tyl RT to read through the proposed branch site was purely conjecture at the outset of our experiments. Our data show that read-through by Ty1 RT on the proposed branch as a template does not in fact occur in vitro with the efficiency necessary to support the observed in vivo st-cDNA synthesis in the absence of Dbrlp debranching activity. Others have studied reverse transcriptases and their ability to read through nonnative linkages in various contexts. For example, both MMLV RT and AMV RT are able to read through linear $2^{\prime}-5^{\prime}$ linkages, albeit with a substantial pause after the nonnative linkage is passed (Lorsch et al. 1995). Because of those results and because various RTs have the ability to read through bona fide $2^{\prime}, 5^{\prime}$-branch sites (Vogel et al. 1997; Tuschl et al. 1998; Vogel and Borner 2002) or other lesions (Cai et al. 1993), initially it seemed acceptable to hypothesize - without direct evidence - that readthrough of the proposed Tyl branch by Tyl RT could be responsible for st-cDNA synthesis in $d b r 1$ cells (Cheng and Menees 2004; Perlman and Boeke 2004). However, our data for the first time directly demonstrate that the proposed Tyl branched RNA cannot be used as an efficient template 
for st-cDNA synthesis by the Tyl RT under in vitro conditions that are known to allow formation of st-cDNA using nonbranched Tyl RNA as template (Cristofari et al. 2002). Therefore, the hypothesis of in vivo branch readthrough by Ty1 RT leading to st-cDNA synthesis in the absence of Dbr1p is now much less tenable. A corollary of our conclusion is that the proposed branch is likely not a Ty1 retrotransposition intermediate, in agreement with the indirect evidence from Coombes and Boeke (2005).

Even with our data, it remains possible that a small amount of 2',5'-branched RNA is formed during Tyl retrotransposition but that this branched RNA is not an intermediate in Tyl cDNA synthesis. Our data do not rule out in vivo formation of branched RNA that is not used as a template for cDNA synthesis. However, even if this were the case, another mechanistic pathway must be responsible for the production of st-cDNA in $d b r l$ cells, for the reasons stated above. The most likely candidate mechanism remains the use of linear $5^{\prime}+3^{\prime}$ Tyl RNAs as the template (Fig. 1A), with the two RNAs held together noncovalently by the CYC base-pairing interaction (Cristofari et al. 2002) or by other secondary structure interactions that have not yet been identified.

If $2^{\prime}, 5^{\prime}$-branched RNA is not a Tyl retrotransposition intermediate that is debranched by Dbrlp, then what is the biological role of this enzyme in retrotransposition? The Dbrlp may simply bind RNA rather than cleave $2^{\prime}, 5^{\prime}$ phosphodiester bonds during Tyl retrotransposition (Salem et al. 2003). Alternatively, Dbr1p could be required in vivo to remove a primer that initiated cDNA synthesis via its 2 '-hydroxyl group, or Dbrlp could have an indirect role via modulating deoxyribonucleotide concentrations (Lauermann et al. 1995; Karst et al. 2000). Although some as yet unidentified protein factor could theoretically facilitate efficient in vivo read-through by Tyl RT of a covalently branched Dbrlp-sensitive RNA intermediate, no available evidence supports this speculation. It is also possible (but without evidence) that some other debranching activity not due to Dbrlp exists in yeast. At present the role in retrotransposition of Dbr1p is still unexplained. The simplest interpretation of our direct evidence, along with the indirect data of Coombes and Boeke (2005), is that Dbrlp does not debranch an obligatory 2',5'-branched RNA linkage during the normal biological pathway of Ty1 retrotransposition.

\section{MATERIALS AND METHODS}

\section{General methods}

All denaturing PAGE was performed with gels prepared using 29:1 acrylamide:bis-acrylamide and $7 \mathrm{M}$ urea, cross-linked by addition of APS and TEMED. Running buffer was $1 \times$ TBE $(89 \mathrm{mM}$ each Tris and boric acid, $2 \mathrm{mM}$ EDTA at $\mathrm{pH}$ 8.3). Dephosphorylation was performed with CIP (Roche). $5^{\prime}-{ }^{32} \mathrm{P}$-Radiolabeling was performed with $\gamma-{ }^{32} \mathrm{P}$-ATP (NEN) and T4 PNK (Fermentas). Stop solution was $80 \%$ formamide, $1 \times \mathrm{TB}, 50 \mathrm{mM}$ EDTA, and $0.025 \%$ each bromophenol blue and xylene cyanol.

\section{tRNA $^{\text {iMet }}$ primer}

The 75-mer tRNA ${ }^{\text {iMet }}$ (with $5^{\prime}$-A replaced by $5^{\prime}-\mathrm{G}$ to allow transcription; Senger et al. 1992) was transcribed using a doublestranded DNA template prepared by PCR, dephosphorylated, $5^{\prime}-{ }^{32} \mathrm{P}$-radiolabeled, and purified by $8 \%$ denaturing PAGE. The DNA oligonucleotides used in the PCR reaction were as follows: forward template, ACGCACGCTGTAATACGACTCACTATAGG CGCCGTGGCGCAGTGGAAGCGCGCAGGGCTCATAACCCTG ATGTCCTCGG; reverse template, TGGTAGCGCCGCTCGGTTT CGATCCGAGGACATCAGGGTTATG; forward primer, ACGC ACGCTGTAATACG; reverse primer, TGGTAGCGCCGCTCGG (T7 RNA polymerase promoter is underlined; overlapping regions are boldface).

\section{Ty1 RNAs}

The 5' and 3' Tyl RNAs were transcribed using T7 RNA polymerase and double-stranded DNA templates derived from plasmid pGTy1-H3 (Boeke et al. 1985). The individual Ty1 RNAs were purified by $6 \%-8 \%$ denaturing PAGE, extracted, and ethanol-precipitated as described (Wang and Silverman 2003a). The primers to prepare the transcription template for the 5' Ty1 RNA were as follows: forward primer, ACGCACGCTGTAATA CGACTCACTATAGAGGAGAACTTCTAGTATATTCTGTATAC; reverse primer, GTTAACATTGGTGGTGGTCT (T7 RNA polymerase promoter is underlined). The primers to prepare the transcription template for the 3' Ty1 RNA were as follows: forward primer, ACGCACGCTGTAATACGACTCACTATAGGT ATGTTGGAATAGAAATCAACTATCAT; reverse primer, CATT GTTGATAAAGGCTATAATATTAGG (T7 RNA polymerase promoter is underlined). The primers to prepare the transcription template for the 3' Tyl RNA with 3'-terminal hairpin extension were as follows: forward primer, same as above; reverse primer, GAACTTCTAGTATATTCTGTTCTGGATGGCTGGAGCATTGTT GATAAAGGCTATAATATTAGG (hairpin extension region is underlined). The primers to prepare the transcription template for the 3' Tyl RNA with 3'-terminal hairpin extension and $20 \mathrm{nt}$ insert adjacent to the branch-site nucleotide were as follows: forward primer, same as above; reverse primer, GAACTTCTAGT ATATTCTGTTCTGGATGGCTGGAGCATTGTTGATAAAGGCTA TAATATTAGG (hairpin extension region is underlined); forward primer for loop insertion between U5824/G5825, TCCTAAATCCT TATCGGACCAAGCAACGACAAGAGGAGAACTTC; reverse primer for loop insertion between U5824/G5825, GAAGTTCTCCTCTTGT CGTTGCTTGGTCCGATAAGGATTTAGGA (loop regions are underlined).

\section{Synthesis of branched RNA}

The 6CE8 deoxyribozyme (Pratico et al. 2005) was used to synthesize branched RNA. A $30 \mu \mathrm{L}$ sample containing $100 \mathrm{pmol}$ of $3^{\prime}$ Tyl RNA with a hairpin extension at the $3^{\prime}$ terminus (nucleotides 5581-5880+hairpin) (Pratico et al. 2005), 200 pmol of 6CE8 deoxyribozyme, $400 \mathrm{pmol}$ of $5^{\prime}$ Tyl RNA (nucleotides 241-820), and $1200 \mathrm{pmol}$ of DNA disruptor for 5' Tyl RNA (complementary to nucleotides 500-529) in $5 \mathrm{mM}$ HEPES $(\mathrm{pH}$ 
7.5), $15 \mathrm{mM} \mathrm{NaCl}$, and $0.1 \mathrm{mM}$ EDTA was annealed by heating at $95^{\circ} \mathrm{C}$ for $3 \mathrm{~min}$ and cooling on ice for $5 \mathrm{~min}$. The reaction volume was increased to $50 \mu \mathrm{L}$ containing final concentrations of $50 \mathrm{mM}$ HEPES (pH 7.5), $150 \mathrm{mM} \mathrm{NaCl}, 2 \mathrm{mM} \mathrm{KCl}$, and $20 \mathrm{mM} \mathrm{MnCl}_{2}$, incubated at $37^{\circ} \mathrm{C}$ for $25 \mathrm{~min}$, and quenched with an equal volume of stop solution. The $915 \mathrm{nt}$ branched RNA containing the 3' Tyl RNA hairpin extension was purified by $5 \%$ denaturing PAGE. The hairpin extension was removed by cleavage with a 10-23 deoxyribozyme as described (50 mM HEPES at pH 7.5, $150 \mathrm{mM} \mathrm{NaCl}$, $2 \mathrm{mM} \mathrm{KCl}, 5 \mathrm{mM} \mathrm{MnCl}_{2}, 37^{\circ} \mathrm{C}, 20 \mathrm{~min}$ ) followed by $5 \%$ denaturing PAGE to provide the desired $880 \mathrm{nt}$ branched RNA product (Pratico et al. 2005). The 900 nt branched RNA containing the $20 \mathrm{nt}$ insert adjacent to the branch site was synthesized in a similar fashion. In both cases the final amount of branched RNA after purification was $\sim 1$ pmol, limited primarily by the low efficiency of extracting these very large RNAs from the polyacrylamide gels (before the extractions, the yield for branch formation was $\sim 28 \%$ and the yield for cleavage by $10-23$ was $\sim 85 \%$ ).

The 6CE8 deoxyribozyme was CCGCGCTAGAACATGGCAC TCAGAGCGCACGGCGAGTACATGAGACTTCC, where the underlined nucleotides at the $5^{\prime}$ and $3^{\prime}$ termini bind to the $5^{\prime}$ Tyl RNA (nucleotides 241-256) and 3' Tyl RNA (nucleotides 5809-5923), respectively. The DNA disruptor oligonucleotide GGTGGTACTGAAGCAGGTTGAGGAGAGGCA was complementary to nucleotides $500-529$ of the $5^{\prime}$ Tyl RNA. The $10-23$ deoxyribozyme to cleave the $3^{\prime}$-terminal hairpin extension was CTGGATGGCTGGAGGGCTAGCTACAACGAATTGTTGATAAA GGCT, where the underlined nucleotides bind to nucleotides 5864-5879 of the 3' Tyl RNA and to nucleotides 1-14 of the hairpin extension, respectively.

\section{Protein expression and purification}

Others have used the 103-mer TYA-1D peptide as the chaperone for cDNA synthesis (Cristofari et al. 2000), but we do not have the means to prepare such a long polypeptide by solid-phase synthesis. Therefore, we overexpressed and purified the full-length TyA chaperone protein (Roth et al. 2000). The BamHI-HindIII restriction fragment of pQETyA (Luschnig and Bachmair 1997) was ligated into the HindIII-BamHI fragment of pET28a (Novagen). The resulting pET28-TyA (encoding a 476 amino acid protein with an $\mathrm{N}$-terminal $\mathrm{His}_{6}$ tag; the original TyA protein is 440 amino acids) was used to transform Escherichia coli BL21 cells, which were grown in LB containing $50 \mu \mathrm{g} / \mathrm{mL}$ kanamycin to $\mathrm{OD}_{600} \approx 0.6$. After induction with $0.4 \mathrm{mM}$ IPTG for $18 \mathrm{~h}$ at $20^{\circ} \mathrm{C}$, cells were pelleted $\left(4000 \mathrm{~g}, 20 \mathrm{~min}, 4^{\circ} \mathrm{C}\right)$ and lysed by sonication on ice in binding buffer $(20 \mathrm{mM}$ Tris at $\mathrm{pH} 7.9,500 \mathrm{mM} \mathrm{NaCl}$, and $10 \%$ glycerol) containing $5 \mathrm{mM}$ imidazole. The lysate was cleared by centrifugation $\left(19,000 \mathrm{~g}, 30 \mathrm{~min}, 4^{\circ} \mathrm{C}\right)$. The TyA protein was purified from the supernatant under nondenaturing conditions by affinity chromatography using Ni-NTA agarose (Qiagen). The $1.0 \mathrm{~mL}$ column was washed with 10 volumes of binding buffer containing $25 \mathrm{mM}$ imidazole. The TyA protein was eluted with binding buffer containing $300 \mathrm{mM}$ imidazole, desalted on a PD-10 column (GE), and concentrated using a Microcon YM-50 filter (Millipore) to a final concentration of $7.9 \mu \mathrm{M}$ by Bradford assay. The wild-type (Wilhelm et al. 2000) and RNase $\mathrm{H}^{-}$(D486S) mutant (Wilhelm et al. 2001) Tyl reverse transcriptases were expressed and purified by Ni-NTA agarose affinity chromatography as described (Wilhelm et al. 2000).

\section{Formation of nucleoprotein complexes and cDNA synthesis (Fig. 2)}

The procedure was adapted from that reported previously (Cristofari et al. 2002). For the cDNA synthesis assays, $30 \mathrm{pmol}$ of the tRNA ${ }^{\text {iMet }}$ in $38 \mu \mathrm{L}$ of water were heated at $90^{\circ} \mathrm{C}$ for $1 \mathrm{~min}$. The volume was adjusted to $40 \mu \mathrm{L}$ containing $0.75 \mu \mathrm{M}$ tRNA ${ }^{\mathrm{iMet}}$, $5 \mathrm{mM}$ Tris- $\mathrm{HCl}$ ( $\mathrm{pH} \mathrm{7.1)}$, and $1 \mathrm{mM} \mathrm{MgCl}_{2}$, and the sample was cooled rapidly on ice (Cristofari et al. 2002). A $15 \mu \mathrm{L}$ sample was prepared containing 50 fmol 5' Tyl RNA, 100 fmol 3' Tyl RNA, $500 \mathrm{fmol}$ folded tRNA ${ }^{\mathrm{iMet}}$, and TyA chaperone protein at 0-0.4 equivalents per nucleotide of RNA in $20 \mathrm{mM}$ Tris- $\mathrm{HCl}$ ( $\mathrm{pH} 7.5)$, $30 \mathrm{mM} \mathrm{NaCl}, 0.2 \mathrm{mM} \mathrm{MgCl}, 0.01 \mathrm{mM} \mathrm{ZnCl}_{2}, 5 \mathrm{mM}$ DTT, and $8 \mathrm{U}$ of Ribolock ribonuclease inhibitor (Fermentas). Alternatively, $50 \mathrm{fmol}$ of branched RNA or only 5' Tyl RNA were included. The sample was incubated at $30^{\circ} \mathrm{C}$ for $15 \mathrm{~min}$ to form the Tyl nucleoprotein complex, as described (Cristofari et al. 2000). Synthesis of cDNA was initiated by addition of 50 pmol of Tyl RT and dNTPs to a final concentration of $250 \mu \mathrm{M}$ each, in a total volume of $25 \mu \mathrm{L}$ containing $20 \mathrm{mM}$ Tris- $\mathrm{HCl}(\mathrm{pH} 7.5), 30 \mathrm{mM}$ $\mathrm{NaCl}$, and $3 \mathrm{mM} \mathrm{MgCl}_{2}$. After incubation at $25^{\circ} \mathrm{C}$ for $60 \mathrm{~min}$, the sample was quenched with $25 \mu \mathrm{L}$ of $1 \%$ SDS and $10 \mathrm{mM}$ EDTA. The analogous experiments of Darlix and coworkers with linear Tyl RNAs as template used a $30 \mathrm{~min}$ incubation (Cristofari et al. 2002). The nucleic acids were isolated by phenol-chloroform extraction, precipitated with ethanol, and dissolved in $10 \mu \mathrm{L}$ of stop solution. The samples were heated at $95^{\circ} \mathrm{C}$ for $2 \mathrm{~min}$ and analyzed by $8 \%$ denaturing PAGE.

Because our experiments used the full-length TyA chaperone protein rather than the TYA1-D polypeptide (see above), we found it necessary to use fivefold lower concentrations of the 5'+3' Tyl RNAs relative to those reported previously for the cDNA synthesis assay (Cristofari et al. 2002). The concentration of tRNA $^{\text {iMet }}$ was not changed. One consequence of the reduced amount of Ty1 RNAs in our experiments is that the absolute amount of cDNA synthesis product is relatively small, and this likely contributes to the relatively low signal-to-noise ratio. This necessitated the use of the PCR approach for quantifying the st-cDNA products, as described below.

\section{cDNA amplification and quantitation by PCR (Fig. 3)}

From the polyacrylamide gel after cDNA synthesis, the regions corresponding to the positions of ss-cDNA and st-cDNA migration were excised, and the nucleic acids were isolated by extraction with $10 \mathrm{mM}$ Tris ( $\mathrm{pH}$ 8.0), $1 \mathrm{mM}$ EDTA, and $300 \mathrm{mM} \mathrm{NaCl}$ followed by ethanol precipitation. For both ss-cDNA and st-cDNA, the cDNA products from two samples (0.1-0.2 TyA:nt ratio) were combined and dissolved in $300 \mu \mathrm{L}$ of water. From each stock sample for the $5^{\prime}+3^{\prime}$ control, $1 / 10,1 / 100$, and $1 / 1000$ dilutions were made; these allow $10 \%, 1 \%$, and $0.1 \%$, respectively, of the st-cDNA product to be used as input into the PCR reaction. The cDNAs were amplified by PCR with appropriate primers; the reverse primer was $5^{\prime}-{ }^{32} \mathrm{P}$-radiolabeled. Each $50 \mu \mathrm{L}$ PCR sample contained $100 \mathrm{pmol}$ forward primer, 25 pmol reverse primer $(0.5$ pmol radiolabeled), $0.2 \mathrm{mM}$ each dNTP, $20 \mathrm{mM}$ Tris- $\mathrm{HCl}(\mathrm{pH}$ 8.8), $10 \mathrm{mM} \mathrm{KCl}, 2 \mathrm{mM} \mathrm{MgSO}$, $10 \mathrm{mM}\left(\mathrm{NH}_{4}\right)_{2} \mathrm{SO}_{4}, 0.1 \%$ Triton $\mathrm{X}-100$, and $2.5 \mathrm{U}$ of Taq polymerase (NEB), along with $30 \mu \mathrm{L}$ of the appropriate dilution of the st-cDNA product. After an initial incubation at $94^{\circ} \mathrm{C}$ for $2 \mathrm{~min}, 30$ cycles of $94^{\circ} \mathrm{C}$ for $1 \mathrm{~min}, 56^{\circ} \mathrm{C}$ 
for $30 \mathrm{sec}$, and $72^{\circ} \mathrm{C}$ for $45 \mathrm{sec}$ were performed, with a final incubation at $72^{\circ} \mathrm{C}$ for $10 \mathrm{~min}$. Of each PCR reaction, $5 \mu \mathrm{L}$ were mixed with $10 \mu \mathrm{L}$ of stop solution and analyzed on $10 \%$ (ss-cDNA) or 6\% (st-cDNA) denaturing PAGE. Band intensities were quantified by PhosphorImager and normalized to 1.0 for PCR from $100 \%$ of the st-cDNA product from the $5^{\prime}+3^{\prime}$ Tyl RNAs. Because the calibration curve constructed from the $5^{\prime}+3^{\prime}$ RNA dilution series is based on PCR and therefore not perfectly linear, the procedure in Figure 3 for determining the amount of st-cDNA produced from the branch is necessary.

The primers for the above experiments were as follows: forward primer for both ss-cDNA and st-cDNA, TGAGAATTGGGTG AATGTTGAGAT (complementary to 5' Tyl RNA nucleotides 311-334); reverse primer for ss-cDNA, GAGGAGAACTTCTAGT ATATTCTG (corresponding to 5' Tyl RNA nucleotides 241-264); reverse primer for st-cDNA, GGTATGTTGGAATAGAAATC AACT (corresponding to 3' Ty1 RNA nucleotides 5581-5604).

To verify the st-cDNA sequence, the $1 / 10$ dilution of the $5^{\prime}+3^{\prime}$ Ty1 RNAs control sample was amplified without radiolabeled primer. The 338 bp PCR product was isolated by $2 \%$ agarose gel electrophoresis and purified by QIAquick extraction (Qiagen). The sequence was confirmed by automated sequencing in both directions using the forward and reverse primers.

\section{ACKNOWLEDGMENTS}

This research was supported by the National Institutes of Health (GM-65966 to S.K.S.). E.D.P. was supported in part by an NIH Cell and Molecular Biology Training Grant fellowship. S.K.S. is the recipient of a fellowship from The David and Lucile Packard Foundation. We thank Yangming Wang, Dana Baum, Claudia Höbartner, and other members of the Silverman laboratory for discussions. We thank J.D. Boeke, A. Bachmair, and A. Gabriel for plasmids as well as J.D. Boeke and S.W. Stevens for comments on the manuscript.

Received May 12, 2007; accepted June 20, 2007.

\section{REFERENCES}

Boeke, J.D., Garfinkel, D.J., Styles, C.A., and Fink, G.R. 1985. Ty elements transpose through an RNA intermediate. Cell 40: 491-500.

Boeke, J.D., Eichinger, D., Castrillon, D., and Fink, G.R. 1988. The Saccharomyces cerevisiae genome contains functional and nonfunctional copies of transposon Ty1. Mol. Cell. Biol. 8: 1432-1442.

Bolton, E.C., Coombes, C., Eby, Y., Cardell, M., and Boeke, J.D. 2005. Identification and characterization of critical cis-acting sequences within the yeast Ty1 retrotransposon. RNA 11: 308-322.

Cai, H., Bloom, L.B., Eritja, R., and Goodman, M.F. 1993. Kinetics of deoxyribonucleotide insertion and extension at abasic template lesions in different sequence contexts using HIV-1 reverse transcriptase. J. Biol. Chem. 268: 23567-23572.

Chapman, K.B. and Boeke, J.D. 1991. Isolation and characterization of the gene encoding yeast debranching enzyme. Cell 65: 483-492.

Cheng, Z. and Menees, T.M. 2004. RNA branching and debranching in the yeast retrovirus-like element Tyl. Science 303: 240-243.

Coombes, C.E. and Boeke, J.D. 2005. An evaluation of detection methods for large lariat RNAs. RNA 11: 323-331.

Coppins, R.L. and Silverman, S.K. 2005. A deoxyribozyme that forms a three-helix-junction complex with its RNA substrates and has general RNA branch-forming activity. J. Am. Chem. Soc. 127: 2900-2907.
Cristofari, G., Ficheux, D., and Darlix, J.L. 2000. The Gag-like protein of the yeast Tyl retrotransposon contains a nucleic acid chaperone domain analogous to retroviral nucleocapsid proteins. J. Biol. Chem. 275: 19210-19217.

Cristofari, G., Bampi, C., Wilhelm, M., Wilhelm, F.X., and Darlix, J.L. 2002. A $5^{\prime}-3^{\prime}$ long-range interaction in Tyl RNA controls its reverse transcription and retrotransposition. EMBO J. 21: 43684379.

Domdey, H., Apostol, B., Lin, R.J., Newman, A., Brody, E., and Abelson, J. 1984. Lariat structures are in vivo intermediates in yeast pre-mRNA splicing. Cell 39: 611-621.

Griffith, J.L., Coleman, L.E., Raymond, A.S., Goodson, S.G., Pittard, W.S., Tsui, C., and Devine, S.E. 2003. Functional genomics reveals relationships between the retrovirus-like Tyl element and its host Saccharomyces cerevisiae. Genetics 164: 867-879.

Havecker, E.R., Gao, X., and Voytas, D.F. 2004. The diversity of LTR retrotransposons. Genome Biol. 5: 225.

Karst, S.M., Rutz, M.L., and Menees, T.M. 2000. The yeast retrotransposons Ty1 and Ty3 require the RNA lariat debranching enzyme, Dbrlp, for efficient accumulation of reverse transcripts. Biochem. Biophys. Res. Commun. 268: 112-117.

Lanciault, C. and Champoux, J.J. 2006. Pausing during reverse transcription increases the rate of retroviral recombination. J. Virol. 80: 2483-2494.

Lauermann, V., Nam, K., Trambley, J., and Boeke, J.D. 1995. Plusstrand strong-stop DNA synthesis in retrotransposon Ty1. J. Virol. 69: 7845-7850.

Lorsch, J.R., Bartel, D.P., and Szostak, J.W. 1995. Reverse transcriptase reads through a $2^{\prime}, 5^{\prime}$-linkage and a $2^{\prime}$-thiophosphate in a template. Nucleic Acids Res. 23: 2811-2814.

Luschnig, C. and Bachmair, A. 1997. RNA packaging of yeast retrotransposon Tyl in the heterologous host, Escherichia coli. Biol. Chem. 378: 39-46.

Mou, Z., Kenny, A.E., and Curcio, M.J. 2006. Hos2 and Set3 promote integration of Ty1 retrotransposons at tRNA genes in Saccharomyces cerevisiae. Genetics 172: 2157-2167.

Perlman, P.S. and Boeke, J.D. 2004. Ring around the retroelement. Science 303: 182-184.

Pratico, E.D., Wang, Y., and Silverman, S.K. 2005. A deoxyribozyme that synthesizes $2^{\prime}, 5^{\prime}$-branched RNA with any branch-site nucleotide. Nucleic Acids Res. 33: 3503-3512.

Roda, R.H., Balakrishnan, M., Kim, J.K., Roques, B.P., Fay, P.J., and Bambara, R.A. 2002. Strand transfer occurs in retroviruses by a pause-initiated two-step mechanism. J. Biol. Chem. 277: 4690046911.

Roda, R.H., Balakrishnan, M., Hanson, M.N., Wohrl, B.M., Le Grice, S.F., Roques, B.P., Gorelick, R.J., and Bambara, R.A. 2003. Role of the reverse transcriptase, nucleocapsid protein, and template structure in the two-step transfer mechanism in retroviral recombination. J. Biol. Chem. 278: 31536-31546.

Rodriguez, J.R., Pikielny, C.W., and Rosbash, M. 1984. In vivo characterization of yeast mRNA processing intermediates. Cell 39: 603-610.

Roth, J.F., Kingsman, S.M., Kingsman, A.J., and Martin-Rendon, E. 2000. Possible regulatory function of the Saccharomyces cerevisiae Ty1 retrotransposon core protein. Yeast 16: 921-932.

Salem, L.A., Boucher, C.L., and Menees, T.M. 2003. Relationship between RNA lariat debranching and Tyl element retrotransposition. J. Virol. 77: 12795-12806.

Senger, B., Despons, L., Walter, P., and Fasiolo, F. 1992. The anticodon triplet is not sufficient to confer methionine acceptance to a transfer RNA. Proc. Natl. Acad. Sci. 89: 10768-10771.

Tuschl, T., Sharp, P.A., and Bartel, D.P. 1998. Selection in vitro of novel ribozymes from a partially randomized U2 and U6 snRNA library. EMBO J. 17: 2637-2650.

Vogel, J. and Borner, T. 2002. Lariat formation and a hydrolytic pathway in plant chloroplast group II intron splicing. EMBO J. 21: 3794-3803. 
Vogel, J., Hess, W.R., and Borner, T. 1997. Precise branch point mapping and quantification of splicing intermediates. Nucleic Acids Res. 25: 2030-2031.

Wang, Y. and Silverman, S.K. 2003a. Characterization of deoxyribozymes that synthesize branched RNA. Biochemistry 42: 15252-15263.

Wang, Y. and Silverman, S.K. 2003b. Deoxyribozymes that synthesize branched and lariat RNA. J. Am. Chem. Soc. 125: 6880-6881.

Wang, Y. and Silverman, S.K. 2005. Efficient one-step synthesis of biologically related lariat RNAs by a deoxyribozyme. Angew. Chem. Int. Ed. Engl. 44: 5863-5866.

Wilhelm, M., Boutabout, M., and Wilhelm, F.X. 2000. Expression of an active form of recombinant Tyl reverse transcriptase in Escherichia coli: A fusion protein containing the C-terminal region of the Tyl integrase linked to the reverse transcriptase-RNase $\mathrm{H}$ domain exhibits polymerase and RNase $\mathrm{H}$ activities. Biochem. J. 38: $337-342$.

Wilhelm, M., Uzun, O., Mules, E.H., Gabriel, A., and Wilhelm, F.X. 2001. Polypurine tract formation by Tyl RNase H. J. Biol. Chem. 276: 47695-47701.

Wu, W., Blumberg, B.M., Fay, P.J., and Bambara, R.A. 1995. Strand transfer mediated by human immunodeficiency virus reverse transcriptase in vitro is promoted by pausing and results in misincorporation. J. Biol. Chem. 270: 325-332.

Zeitlin, S. and Efstratiadis, A. 1984. In vivo splicing products of the rabbit $\beta$-globin pre-mRNA. Cell 39: 589-602.

Zelin, E., Wang, Y., and Silverman, S.K. 2006. Adenosine is inherently favored as the branch-site RNA nucleotide in a structural context that resembles natural RNA splicing. Biochemistry 45: 2767-2771. 

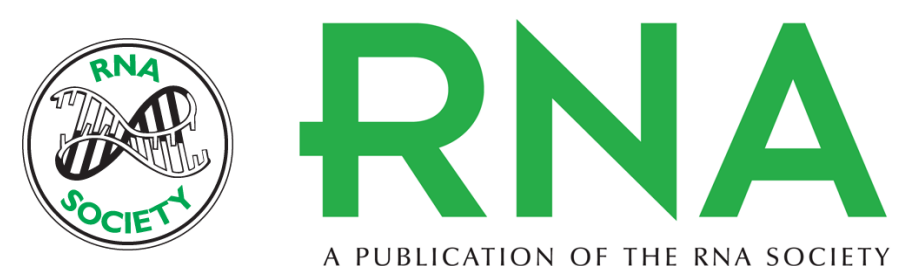

A PUBLICATION OF THE RNA SOCIETY

\title{
Ty1 reverse transcriptase does not read through the proposed $2^{\prime}, 5^{\prime}$ -branched retrotransposition intermediate in vitro
}

\author{
Elizabeth D. Pratico and Scott K. Silverman
}

RNA 2007 13: 1528-1536 originally published online July 24, 2007

Access the most recent version at doi:10.1261/rna.629607

\section{References This article cites 38 articles, 19 of which can be accessed free at: http://rnajournal.cshlp.org/content/13/9/1528.full.html\#ref-list-1}

License
Email Alerting Receive free email alerts when new articles cite this article - sign up in the box at the Service top right corner of the article or click here.

